\title{
Espumados Magnetizáveis Úteis em Processos de Recuperação Ambiental
}

\author{
Magnovaldo C. Lopes, Fernando G. de Souza Jr. \\ Instituto de Macromoléculas Professora Eloisa Mano, UFRJ \\ Geiza E. Oliveira \\ Departamento de Química, UFES
}

\begin{abstract}
Resumo: A produção de biodiesel foi incentivada pelo desejo de se obter fontes energéticas de origem diferente do petróleo. Este processo, contudo, gera grandes quantidades de glicerina como subproduto. Assim, novas aplicações devem ser encontradas para esta glicerina evitando o colapso da cadeia nacional produtiva de glicerina animal. Uma aplicação mais nobre para esta glicerina, proveniente da indústria de biodiesel, é a produção de resinas alquídicas. Estas resinas podem ser utilizadas como matrizes de compósitos para recuperação de ambientes aquáticos impactados por acidentes de derrames de petróleo. Outra aplicação para estes materiais é na remoção e limpeza de águas produzidas e águas de refinaria, visando a atender a legislação ambiental. Neste trabalho, nanocompósitos espumados magnetizáveis foram preparados pela inserção de nanopartículas magnetizáveis em uma matriz de resina alquídica, obtida a partir da glicerina oriunda do óleo de mamona. Os materiais produzidos foram caracterizados por FTIR-ATR, DRX e SAXS. Além disso, a força magnética destes materiais foi estudada bem como a capacidade de remoção de petróleo derramado sobre água. Os resultados são animadores, pois demonstram a obtenção de um nanocompósito que possui força magnética relativa superior à da maguemita pura. Além disso, este nanocompósito é capaz de remover massas de petróleo cerca de 300\% superiores às massas do material usado. Estes materiais, portanto, consistem em uma potencial ferramenta para a recuperação de ambientes aquáticos impactados por acidentes de derramamento de petróleo.
\end{abstract}

Palavras-chave: Poliuretanos, biopolímeros, nanopartículas magnéticas, óleo de mamona.

\section{Magnetic Foams Useful in the Environmental Recovery Processes}

\begin{abstract}
The biodiesel production was encouraged since the entire world is looking for new energy sources. However, the biodiesel process produces large amounts of glycerin as byproduct. Therefore, innovative uses for this new glycerin source must be sought so as to avoid the collapse of the animal glycerin chain. In this context, the production of resins is interesting due to the likeness between these polymers and the petroleum, with these resins being promising as spill cleanup agents. In the present work, magnetic foams were prepared with insertion of maghemite nanoparticles into in a polymer generated from the glycerin, and were characterized using FTIR-ATR, WAXD and SAXS techniques. In addition, the magnetic force and the oil removal capability of these materials were also studied. The results are encouraging because nanocomposites were obtained which possess magnetic forces larger than for pure maghemite. Furthermore, this nanocomposite is able to remove a petroleum mass around 300\% larger than the used mass of the composite, which means that the composite may be important for avoiding environmental disasters owing to the oil spill on the water.
\end{abstract}

Keywords: Polyurethanes, biopolymers, magnetic nanoparticles, castor oil.

\section{Introdução}

Atualmente há uma enorme preocupação mundial quanto ao uso sustentável dos recursos naturais. A enorme demanda pelo petróleo poderá acarretar em sua escassez. Além disso, a extração e produção de petróleo são atividades de alto risco ambiental. Estas características tornam necessário o desenvolvimento imediato de fontes de matérias primas alternativas para as próximas gerações ${ }^{[1]}$. Porém, a produção de polímeros a partir de recursos renováveis geralmente apresenta custo elevado e baixos rendimentos. Desta forma, a procura por materiais renováveis que possam ser polimerizados e que apresentem alto rendimento deve ser encorajada ${ }^{[2]}$.

As resinas de poliuretano (PU) podem ser derivadas tanto do petróleo quanto de fontes naturais, como os óleos vegetais. Estes óleos são polióis com uma ampla distribuição de peso molecular e um considerável grau de ramificação. Estas características afetam as propriedades, como por exemplo, a viscosidade dos poliuretanos
(PUs) produzidos a partir deles. Os polióis de origem renovável constituem excelentes fontes de matérias-primas renováveis para a fabricação de PUs ${ }^{[3,4]}$. Entre estes, o óleo de mamona vem se destacando por apresentar uma composição química centrada em um alto teor de ácido ricinoleico. Este ácido possui grupos hidroxila e duplas ligações que o tornam adequado para muitas reações químicas $^{[4]}$, entre as quais merece destaque a transesterificação que leva ao biodiese ${ }^{[5]}$.

Devido à elevada reatividade dos isocianatos, que conduz a uma variedade de reações possíveis, os PUs podem apresentar propriedades mecânicas e morfológicas diversas ${ }^{[6]}$. Esta diversidade de propriedades permite que os Pus possam ser aplicados em vários tipos de industrias, tais como: (i) na indústria automobilística (volantes, painéis, assentos e para-choques), (ii) na fabricação de móveis (colchões e assentos), (iii) na indústria de calçados (solados de sapatos e adesivos), (iv) na indústria de eletrodomésticos (refrigeradores e aquecedores), (v) entre outros ${ }^{[7]}$. 
Mesmo com toda a versatilidade e boas características, diversas propriedades dos PUs podem ser melhoradas com o uso de cargas minerais ${ }^{[8]}$. O uso destas partículas inorgânicas, de tamanho nanométrico, incorporadas à matriz polimérica vem despertando grande interesse industrial e científico ${ }^{[9,10]}$. Este interesse está relacionado com a obtenção de propriedades superiores, como por exemplo, nos casos da barreira a gases, módulo de elasticidade, resistência à tração, resistência a solventes e ao fogo, entre outras ${ }^{[8]}$. Essa melhora nas propriedades é obtida com a incorporação de pequenos teores da carga inorgânica, geralmente na faixa de 1 a $10 \%$ em massa ${ }^{[11,12]}$. A melhoria das propriedades físicas do compósito está relacionada com as dimensões nanométricas das cargas, que produzem uma área superficial muito elevada, responsável por uma melhor interação com a matriz polimérica ${ }^{[13]}$.

Entre as nanopartículas, os óxidos de ferro são os materiais magnéticos mais estudados. A incorporação destas nanopartículas em matrizes poliméricas é devido as suas características e propriedades favoráveis como: (i) menor custo de produção, (ii) menor densidade em comparação com partículas metálicas, (ii) facilidade de preparo de nanopartículas com tamanho e distribuição controlada e (iv) elevada biocompatibilidade ${ }^{[14,15]}$.

Além da característica magnética, estes compósitos apresentam semelhança química com o petróleo (constituído principalmente por hidrocarbonetos saturados, hidrocarbonetos aromáticos, resinas e asfaltenos $\left.{ }^{[16-19]}\right)$. Esta semelhança química torna estes materiais potenciais removedores de petróleo da água. Este material pode ser usado tanto no caso de derramamentos de petróleo em ambiente aquático (acidentes ecológicos) quanto para limpeza de águas produzidas e águas de refinaria. Estas águas só podem ser descartadas com concentração máxima de $29 \mathrm{mg}$ de petróleo por litro de água, segundo a Resolução no 393/2007 do Conama ${ }^{[20]}$.

Assim, o presente trabalho busca obter resinas magnetizáveis, pela incorporação de nanopartículas de maghemita em poliuretanos. Estas resinas são úteis em processos de recuperação ambiental focados na remoção de petróleo de ambientes aquáticos. Os materiais produzidos foram caracterizados por microscopia óptica (MO), espectroscopia de infravermelho com transformada de Fourrier (FTIR), difração de raios X (DRX) e por espalhamento de raios X a baixo ângulo (SAXS). Além disso, a força magnética e a capacidade de remoção de petróleo destes materiais também foram estudadas. Este estudo permitiu estabelecer um modelo empírico capaz de descrever a relação entre a massa de resina usada e a quantidade de óleo removida da superfície da água.

\section{Experimental}

\section{Materiais}

Cloreto férrico $\left(\mathrm{FeCl}_{3}\right)$, sulfito de sódio $\left(\mathrm{Na}_{2} \mathrm{SO}_{3}\right)$ e hidróxido de amônio $\left(\mathrm{NH}_{4} \mathrm{OH}\right)$ foram fornecidos pela Vetec (Rio de Janeiro, Brasil). O óleo de mamona, grau comercial, foi fornecido pela Campestre Ind. e Com. de Óleos Vegetais LTDA (São Paulo, Brasil). O diisocianato de tolueno, grau comercial, foi adquirido da Epoxifibers Comércio de Produtos Químicos (Rio de Janeiro, Brasil). Todos os reagentes foram utilizados como recebidos.

\section{Sintese de maghemita}

A maghemita foi preparada por co-precipitação homogênea, seguindo o procedimento descrito por Qu e colaboradores ${ }^{[21]}$. Em um procedimento típico, $30 \mathrm{~mL}$ de solução de $\mathrm{FeCl}_{3}(2 \mathrm{M}), 20 \mathrm{~mL}$ de Solução de $\mathrm{Na}_{2} \mathrm{SO}_{3}(1 \mathrm{M})$ e $\mathrm{NH}_{4} \mathrm{OH}$ concentrado $(1,4 \mathrm{M})$ foram dissolvidos em $900 \mathrm{~mL}$ de água. Após a mistura dos reagentes, surgiu um precipitado de coloração escura. O sobrenadante foi descartado e o precipitado foi lavado com água destilada. O procedimento foi repetido várias vezes até $\mathrm{pH} 7 . \mathrm{O}$ precipitado foi filtrado e seco à temperatura ambiente. Em seguida, a fim de converter magnetita em maghemita, o precipitado foi submetido a tratamento térmico a $200{ }^{\circ} \mathrm{C}$ durante 1 hora.

\section{Síntese do poliuretano}

O óleo de mamona, o diisocianato de tolueno e a água destilada foram vertidos em um béquer e homogeneizados por 120 segundos com o auxílio de um sonicador (UNIQUE Modelo DES500). Foram preparadas 4 amostras de espumados de PU, com diferentes volumes de água e diisocianato, conforme apresentados na Tabela 1.

\section{Caracterização}

Os experimentos de espalhamento de raios X a baixo ângulo das partículas de maghemita foram feitos nalinha de SAXS doLaboratório Nacional de Luz Síncrotron ${ }^{[22]}$. Esta linha de luz é equipada com um monocromador de corte assimétrico $(\lambda=1.743 \AA)$, horizontal ao feixe da luz. Um detector sensível a raios X (PSD) e um analisador multicanal foram usados para determinar a intensidade SAXS, I(q), em função do modulo do vetor de espalhamento $q=(4 \pi / \lambda) \sin \theta$, onde $2 \theta$ é o ângulo de espalhamento. Todos os espalhamentos tiveram o espalhamento parasita e a não homogeneidade do detector corrigidos $^{[23]}$.

As análises de infravermelho foram realizadas em equipamento Thermo Nicolet iN10, com acessório de reflexão total atenuada (ATR) equipado com cristal de seleneto de zinco. As amostras foram, previamente, secas e acondicionadas em um dessecador. As análises de infravermelho foram realizadas com acumulo de 100 varreduras e com resolução de $4 \mathrm{~cm}^{-1}$.

Após a polimerização, o volume interno dos poros abertos das amostras de PU foi determinado pela técnica de imersão em água. Em seguida, com o auxílio de um microscópio Bel Photonics, o diâmetro dos poros abertos foi determinado. Os dados de diâmetro foram usados para a construção de curvas de distribuição de probabilidade seguindo metodologia descrita em trabalhos anteriores do grupo ${ }^{[24]}$.

A cristalinidade das amostras foi determinada por difração de raios X usando um Difratômetro Miniflex da Rigaku, com resolução de $0,05^{\circ}$ no intervalo de $2 \theta$ entre $1^{\circ}$ e $70^{\circ}$.

As amostras pulverizadas foram submetidas a um ensaio de força magnética. Para tanto se utilizou uma balança analítica Shimadzu AY-220, um imã de Nd campo magnético igual a $(0,419 \pm 0,001) \mathrm{T}$ (determinado com o auxílio de um Gausímetro GlobalMag modelo TLMP-Hall-10) e um paquímetro. O teste consiste em medir a massa do compósito em dois momentos distintos, na ausência do campo magnético e na presença do campo magnético. A variação de massa, obtida por subtração da massa da amostra na ausência e na presença do campo magnético, é relacionada com a força magnética considerando a distância da amostra até a metade do imã. $\mathrm{O}$ ensaio é baseado no método descrito por Davis ${ }^{[25]}$. A força magnética é calculada de acordo com a Equação1:

$$
F m=\Delta m \times g
$$

Tabela 1. Composições usadas para o preparo das amostras de PU.

\begin{tabular}{cccc}
\hline Amostra & $\begin{array}{c}\text { Óleo de mamona } \\
(\mathbf{m L})\end{array}$ & $\begin{array}{c}\text { Diisocianato } \\
(\mathbf{m L})\end{array}$ & $\begin{array}{c}\text { Água } \\
(\mathbf{m L})\end{array}$ \\
\hline PU-1 & 2,0 & 2,0 & 0,25 \\
PU-2 & 2,0 & 2,0 & 0,35 \\
PU-3 & 2,0 & 2,0 & 0,45 \\
PU-4 & 2,0 & 1,0 & 0,25 \\
\hline
\end{tabular}


Na Equação 1, Fm é a força magnética; $\Delta m$ é a variação de massa na presença do campo magnético e $g$ é a aceleração da gravidade.

Para os testes de remoção de petróleo, uma massa conhecida da resina magnetizável foi pesada em vidro de relógio e reservada. Na etapa seguinte, $90 \mathrm{~mL}$ de água salina foram adicionados a um béquer de $100 \mathrm{~mL}$. Sobre a água foram adicionados $10 \mathrm{~g}$ de petróleo, seguido da adição da massa conhecida da resina, sobre o petróleo. Aguardaram-se 5 minutos e então um imã de $\mathrm{Nd}$ com campo magnético igual a $(0,419 \pm 0,001) \mathrm{T}$ foi aproximado da mistura de petróleo e resina, removendo parte dessa massa do sistema. Ao final do processo, a massa de petróleo removida foi determinada por gravimetria.

\section{Resultados}

As nanopartículas de maghemita foram escolhidas para a incorporação no poliuretano por apresentarem um bom comportamento magnético. Este comportamento corresponde à magnetização residual próxima de zero após a remoção do campo magnético aplicado ${ }^{[26]}$, ilustrado na Figura 1. Esta figura apresenta o material particulado na presença de um campo magnético (Figura 1a) e fora da ação deste campo (Figura 1b), evidenciando que a presença do imã provoca um alinhamento do material. Esse alinhamento é facilmente desfeito após a remoção do magneto.

A Figura 2 apresenta os espectros das resinas alquídicas reticuladas com diferentes quantidades de diisocianato de tolueno e água. Pode-se observar que, de uma maneira geral, todos os espectros apresentam as mesmas bandas características e que a amostra PU-4 apresenta as bandas mais intensas. Pode-se observar uma banda larga, de baixa intensidade, ao redor de $3300 \mathrm{~cm}^{-1}$ característica da deformação axial de ligações de hidrogênio dos grupos NH. O dublete observado na região de $2900 \mathrm{~cm}^{-1}$ corresponde à deformação axial da ligação $\mathrm{C}-\mathrm{H}$ dos grupos $\mathrm{CH}_{2}$ e $\mathrm{CH}_{3}$. A banda característica intensa observada em $1727 \mathrm{~cm}^{-1}$ está relacionada a deformação axial da ligação $\mathrm{C}=\mathrm{O}$ conjugada com banda de amida I. As bandas características que aparecem em 1600 e $1512 \mathrm{~cm}^{-1}$ são devidas às deformações angulares das ligações $\mathrm{N}-\mathrm{H}$, chamadas de banda de amida II. A banda característica que aparece em $1411 \mathrm{~cm}^{-1}$ corresponde à deformação axial da ligação C-N. A banda ao redor de $1300 \mathrm{~cm}^{-1}$ está relacionada à interação entre a deformação angular da ligação N-H e a deformação axial da ligação C-N. A banda observada em $1215 \mathrm{~cm}^{-1}$ é característica da deformação axial da ligação $(\mathrm{C}=\mathrm{O})-\mathrm{O}$. A banda característica ao redor de $1045 \mathrm{~cm}^{-1}$ corresponde à deformação axial da ligação C-O-C. Já a banda característica na região ao redor de $700 \mathrm{~cm}^{-1}$, banda larga, está relacionada à deformação angular fora do plano da ligação N-H.

A micro-estrutura desses compósitos foi avaliada por DRX e por SAXS. A difração de raios X das resinas, do compósito contendo 5\% de maghemita (PU-5) e da maghemita pura é mostrada na Figura 3.

A comparação entre os difratogramas do compósito (PU-5) e da maghemita pura permite inferir que a estrutura das partículas magnéticas não é alterada pela fase polimérica (Figura 3 e Tabela 2). As partículas de maghemita apresentam uma estrutura maciça ${ }^{[24]}$, sendo incapazes de sofrer esfoliação quando misturadas na matriz. Isto é evidenciado pela ausência de mudanças dos valores de espaçamento interplanar (d) e de tamanho do cristalito (Lc), calculados usando as equações de Bragg e Scherrer, respectivamente ${ }^{[27]}$.

Os valores de cristalinidade, apresentados na Tabela 2, foram calculados seguindo o método de Ruland ${ }^{[28]}$. Estes valores mostraram que, independentemente do modo de preparo utilizado, as amostras das resinas puras apresentaram baixos valores de cristalinidade e estatisticamente iguais.

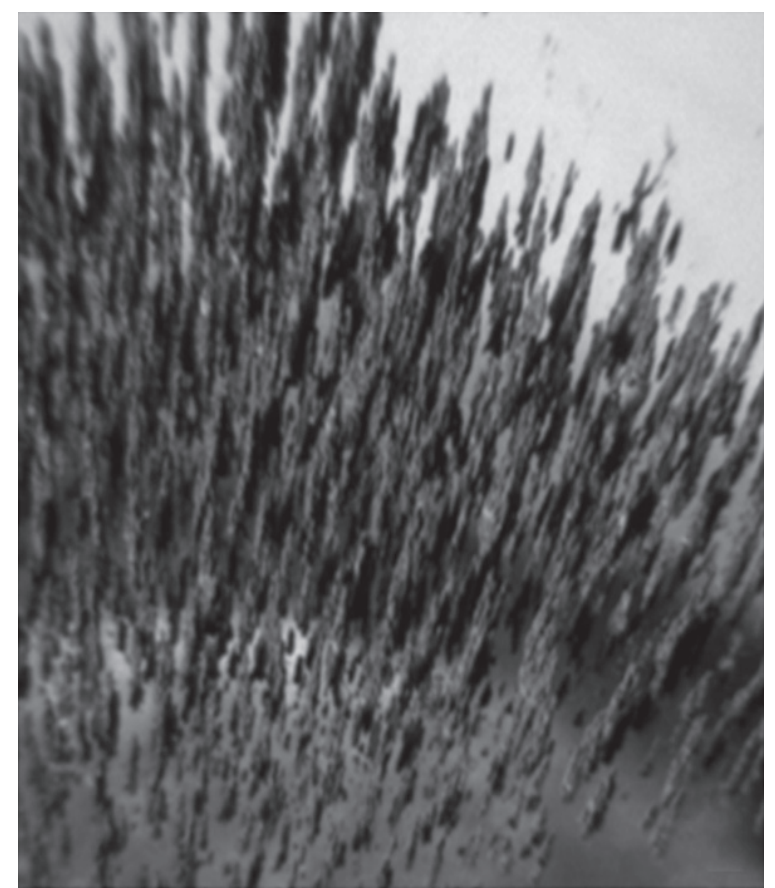

(a)

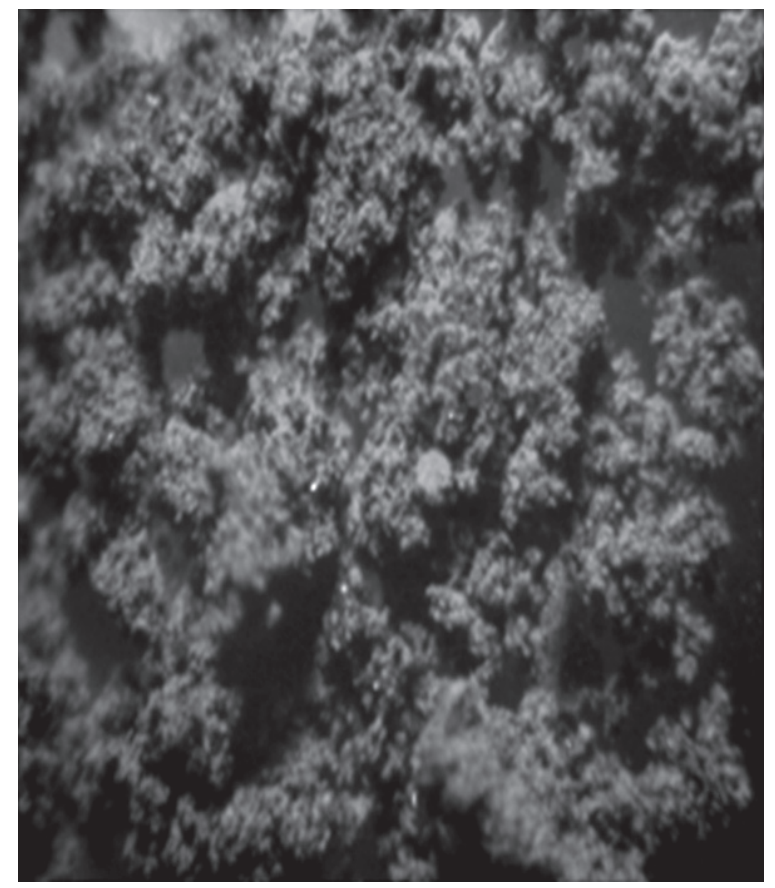

(b)

Figura 1. Partículas de maghemita sob (a) e fora (b) da influência de um campo magnético.

Este resultado é bastante interessante, uma vez que diferentes graus de cristalinidade poderiam implicar em diferentes distribuições de densidade no interior da matriz. Entre as amostras estudadas, conforme o esperado, apenas a amostra preenchida com $5 \%$ de maghemita apresentou significativa mudança da cristalinidade.

A caracterização por SAXS do compósito magnetizável permitiu, via a integração de setor seguida das correções apropriadas, construir o gráfico apresentado na Figura 4. Por sua vez, a deconvolução gaussiana destes dados permitiu o cálculo dos dados mostrados na Tabela 3. 


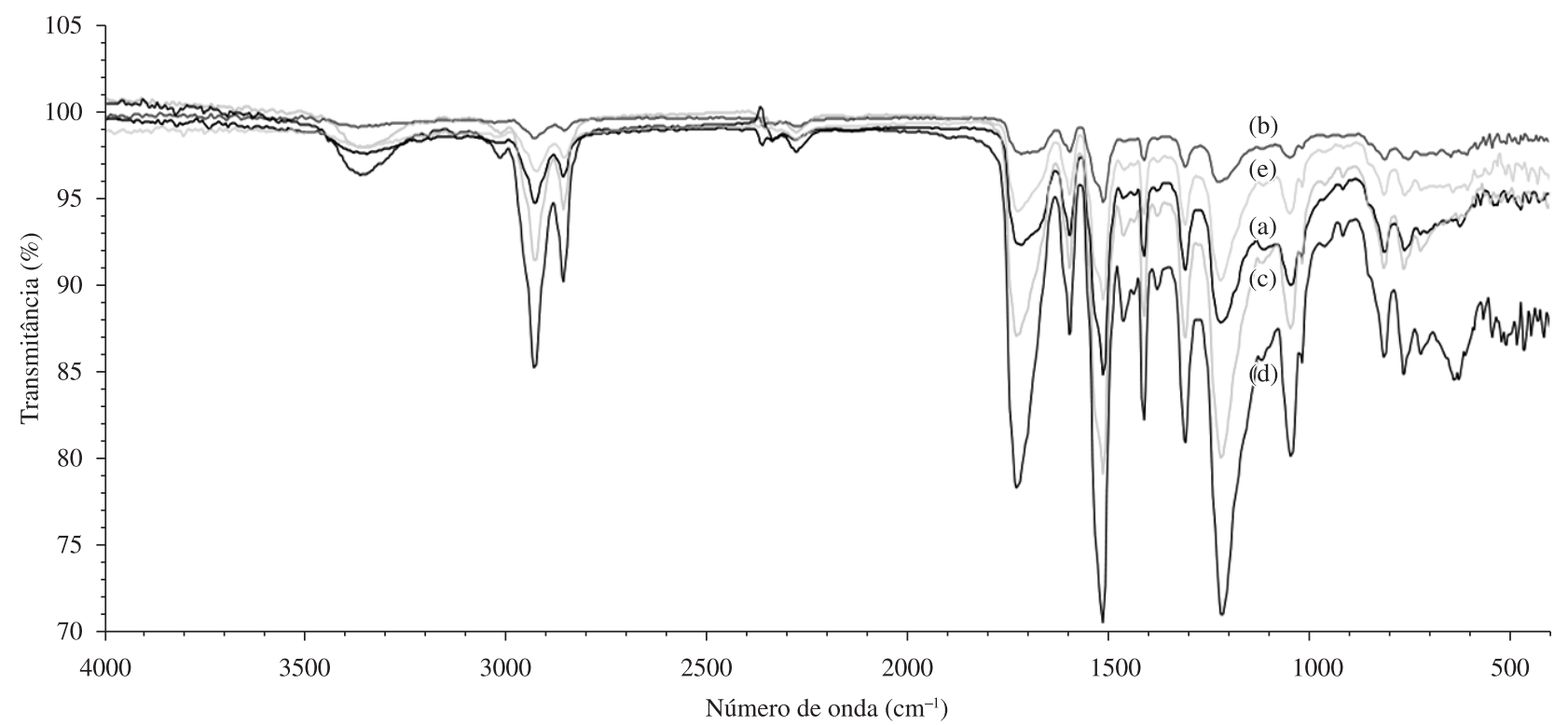

Figura 2. FTIR das amostras PU-1 (a); PU-2 (b); PU-3 (c); PU-4 (d); e PU-5 (e).

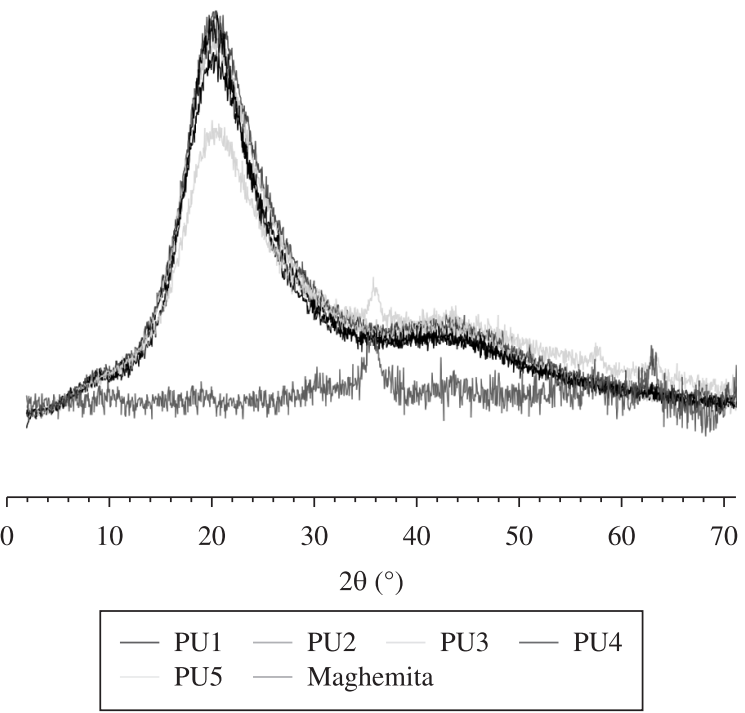

Figura 3. Difratogramas das resinas (PU1-PU4), do compósito (PU5) e da maghemita.

Tabela 2. Cristalinidade das amostras de poliuretanos com respectivos desvios padrão.

\begin{tabular}{cccc}
\hline Amostra & $\begin{array}{c}\text { Cristalinidade } \\
(\boldsymbol{\%})\end{array}$ & $\begin{array}{c}\mathbf{d} \\
(\mathbf{n m})^{*}\end{array}$ & $\begin{array}{c}\text { Lc } \\
(\mathbf{n m})^{* *}\end{array}$ \\
\hline PU-1 & $9 \pm 2$ & - & - \\
PU-2 & $12 \pm 1$ & - & - \\
PU-3 & $13 \pm 1$ & - & - \\
PU-4 & $12 \pm 4$ & - & - \\
PU-5 & $21 \pm 3$ & $0,251 \pm 0,003$ & $21 \pm 2$ \\
maghemita & $70 \pm 1$ & $0,250 \pm 0,002$ & $17 \pm 2$ \\
\hline
\end{tabular}

*Espaçamento interplanar e **tamanho do cristalito.

Tabela 3. Principais resultados obtidos do SAXS.

\begin{tabular}{ccccc}
\hline Pico & $\begin{array}{c}\text { Centro } \\
\left(\mathbf{n m}^{-1}\right)\end{array}$ & $\begin{array}{c}\mathbf{F W H M} \\
\left(\mathbf{n m}^{-1}\right)\end{array}$ & $\begin{array}{c}\mathbf{d s} \\
(\mathbf{n m})\end{array}$ & $\begin{array}{c}\mathbf{L c} \\
(\mathbf{n m})\end{array}$ \\
\hline 1 & 0,27 & 0,21 & $23,3 \pm 0,2$ & $59,8 \pm 0,6$ \\
2 & 0,42 & 0,33 & $15,0 \pm 0,1$ & $38,1 \pm 0,4$ \\
\hline
\end{tabular}

Os resultados das análises de SAXS (Figura 4) mostram a presença de dois tipos principais de centros espalhantes. O primeiro deles é constituído por partículas de aproximadamente $60 \mathrm{~nm}$ de diâmetro, separadas por uma região de menor densidade eletrônica de aproximadamente $23 \mathrm{~nm}$. Já o segundo é formado por partículas de cerca de $38 \mathrm{~nm}$ de diâmetro, separadas por uma distância de aproximadamente $15 \mathrm{~nm}$.

Os resultados de AFM de outro trabalho do grupo ${ }^{[29]}$ mostraram que as partículas de maghemita sintetizadas segundo o método aqui descrito possuem, com $95 \%$ de probabilidade, um aspecto esférico de diâmetro igual a $28_{-14}^{+18} \mathrm{~nm}$. Já os resultados de difração de raios $X$ indicam tamanho de cristalito igual a $(17 \pm 2) \mathrm{nm}$ e $(21 \pm 2) \mathrm{nm}$ para a maghemita pura e para a maghemita presente no compósito PU-5, respectivamente. Estes resultados estão em pleno acordo com outros reportados por Nedkova et al. ${ }^{[30]}$.

Por sua vez, os resultados de SAXS (Figura 4) mostram a existência de centros espalhantes no interior da matriz do compósito PU-5 de diâmetros iguais a $(38.1 \pm 0.4) \mathrm{nm}$ e $(59.8 \pm 0.6) \mathrm{nm}$. Considerando que o menor valor de tamanho de partícula determinado por AFM $(\sim 14 \mathrm{~nm})$ corresponde ao tamanho dos monodomínios de maghemita, é possível concluir que há uma boa concordância entre essa informação e a obtida por difração de raios $\mathrm{X}$. Por outro lado, todos estes valores são substancialmente maiores que os observados por SAXS. O volume calculado a partir dos dados de difração da amostra de maghemita é igual a aproximadamente $7700 \mathrm{~nm}^{3}$. Já os volumes calculados a partir dos dados de SAXS do compósito PU-5 são iguais a 86760 e $336650 \mathrm{~nm}^{3}$. Assim, os centros espalhantes presentes no compósito PU-5 parecem ser formados de aglomerados de cerca de 11 e 44 monodomínios de maghemita. Embora estes resultados evidenciem algum grau de agregação das nanopartículas de maghemita no compósito, isso não descaracteriza a natureza nanoscópica dos materiais produzidos, os quais podem ser classificados como nanocompósitos.

Os resultados de imersão em água são mostrados na Tabela 4. Todos os testes foram feitos a $30{ }^{\circ} \mathrm{C}$ e em triplicata. Os resultados de imersão mostram que, entre os materiais preparados, o aumento da quantidade de água na composição possivelmente provocou um enfraquecimento das paredes, evidenciado pela diminuição contínua da porosidade das amostras PU-1 a PU-4. Este enfraquecimento é causado pela diminuição do diisocianato disponível para a reação com as hidroxilas do óleo de mamona. Isso produziu o colapso de várias células, o que levou à diminuição do volume interno. 


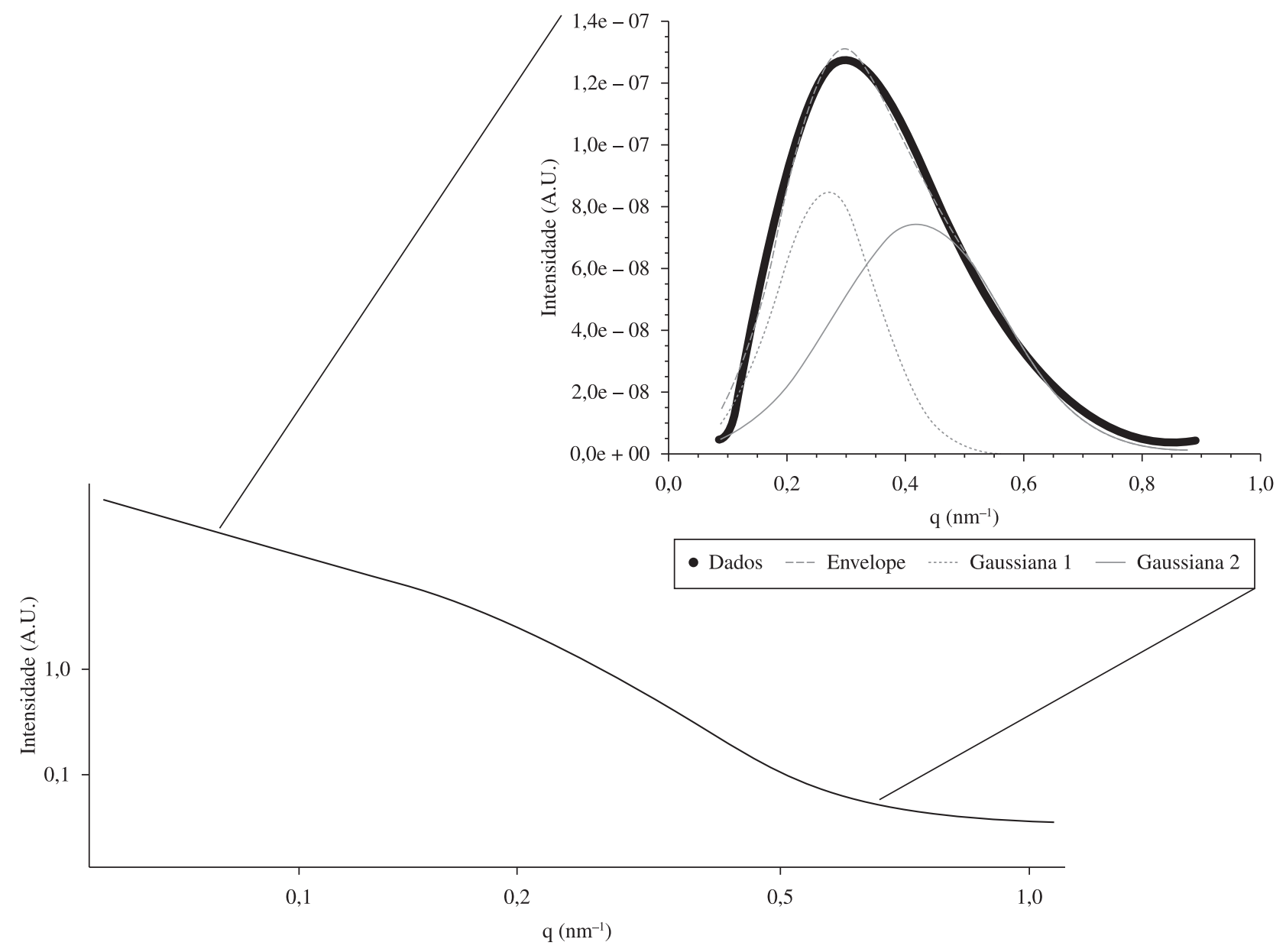

Figura 4. SAXS da maghemita incorporada no compósito e detalhe da deconvolução gaussiana.

Tabela 4. Volume dos poros abertos das amostras de poliuretanos.

\begin{tabular}{cc}
\hline Amostra & Volume $\left(\mathbf{c m}^{\mathbf{3}}\right)$ \\
\hline PU-1 & $2,6 \pm 0,2$ \\
PU-2 & $2,0 \pm 0,2$ \\
PU-3 & $1,2 \pm 0,1$ \\
PU-4 & $0,7 \pm 0,1$ \\
PU-5 & $2,0 \pm 0,2$ \\
\hline
\end{tabular}

Os resultados da Tabela 4 mostram que a amostra PU-1 (preparada com $0,25 \mathrm{~mL}$ de água e 2,0 $\mathrm{mL}$ de diisocianato) foi o material que absorveu a maior massa de líquido. $\mathrm{O}$ volume interno dos poros abertos deste material é igual a $2,6 \pm 0,2 \mathrm{~cm}^{3}$. Essa informação é valiosa, permitindo inferir que esse material é composto por paredes mais finas, ideais para o preparo do material particulado necessário ao processo de recuperação ambiental aqui estudado. Por isso a condição de preparo das amostras PU-1 foi à escolhida para o preparo do compósito com as nanopartículas de maghemita. Este material contendo as nanopartículas foi denominado. As amostras de PU-5 apresentaram uma diminuição do volume interno de poros maior que a da formulação PU-1 e similar a da formulação PU-2 (Tabela 4). Esta diminuição do volume interno do PU-5 está, possivelmente, relacionada com o aumento da massa das paredes. Este aumento da massa das paredes resultou em maior grau de colapso. Contudo, mesmo com paredes mais espessas, a amostra PU-5 apresentou facilidade de maceração similar ao do PU-1.
A morfologia das amostras foi estudada por microscopia ótica. As micrografias obtidas são mostradas na Figura 5. Os resultados morfológicos mostram que a amostra PU-1 apresenta grandes poros conectados, a maioria de aspecto aberto. Já a amostra PU-2 também apresenta grandes poros, contudo é possível observar que estes poros são menores e as paredes mais espessas. Esse comportamento de diminuição dos poros e aumento das paredes se torna mais visível à medida que mais água é utilizada, conforme observado nas amostras PU-3 e PU-4. Estes resultados estão em acordo com os resultados do volume interno, sustentando a discussão apresentada. De maneira semelhante, a amostra PU-5 (Figura 5c e 5d) apresentou poros menores que os apresentados por seu análogo PU-1 possivelmente devido ao já discutido aumento da massa das paredes, que provoca certo colapso. Além disso, o nanocompósito PU-5 apresentou coloração mais avermelhada ocasionada pela presença de maghemita.

Entre os materiais preparados, o compósito contendo 5\% de maghemita (PU-5) e a maghemita pura foram submetidos ao ensaio de força magnética. Como ilustração, os resultados de força magnética em função da distância entre a amostra e o magneto do compósito contendo 5\% de maghemita são mostrados na Figura 6.

O teste consiste em medir a massa do compósito em dois momentos distintos: uma sem a presença do campo magnético e outra na presença do campo magnético posicionado a uma distância conhecida da amostra. Observa-se um aumento exponencial na força magnética na medida em que o magneto é aproximado das amostras do compósito e de maghemita. O modelo empírico que descreve este comportamento é mostrado na Equação 2. 


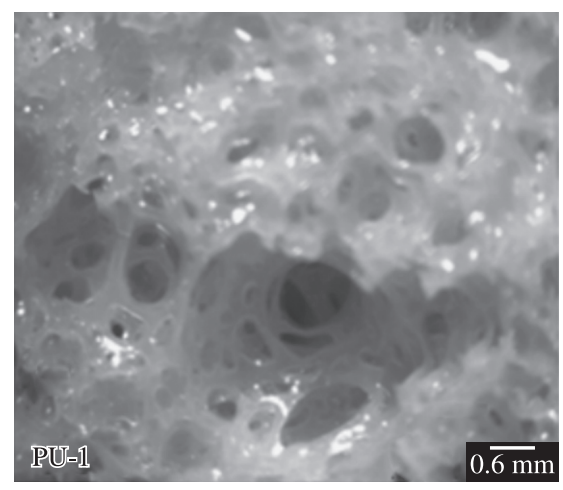

(a)

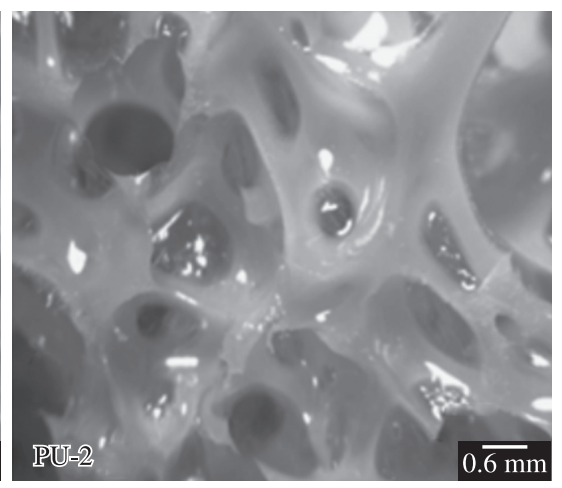

(b)

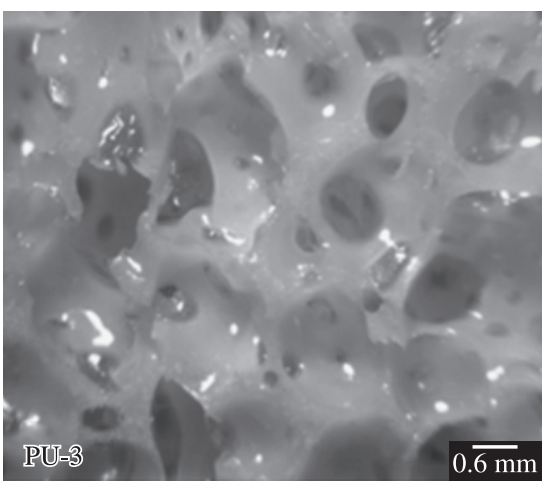

(c)

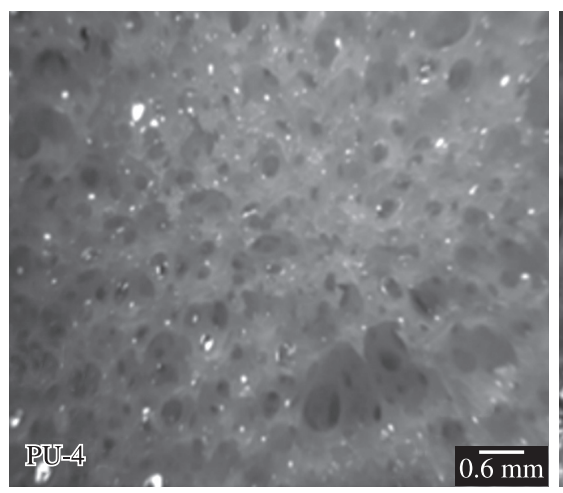

(d)

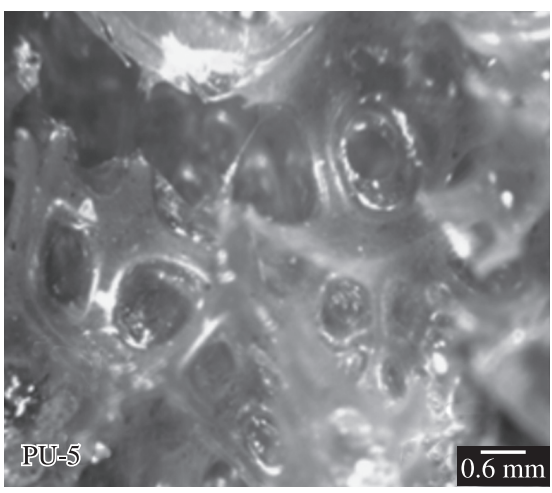

(e)

Figura 5. Resinas de poliuretano: PU-1 (a); PU-2 (b); PU-3 (c); PU-4 (d); e PU-5 (e).

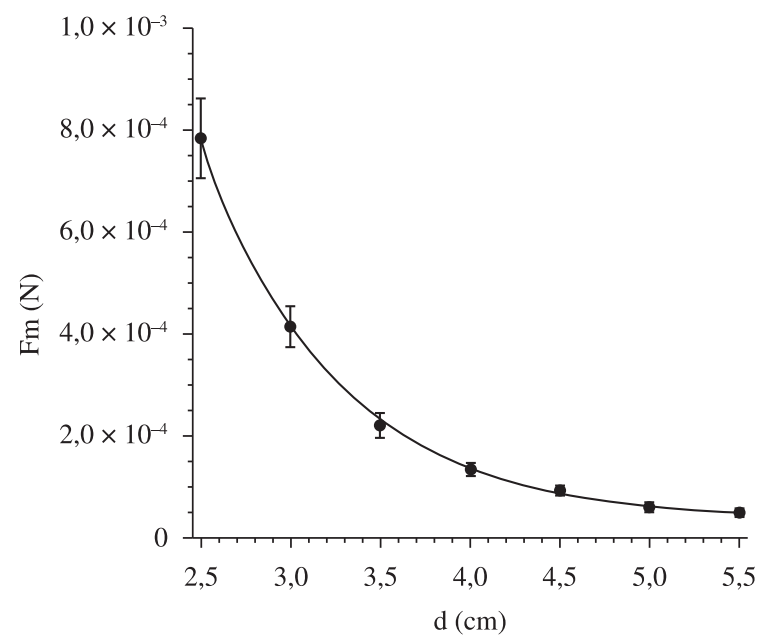

Figura 6. Variação da força magnética em função da distância entre a amostra e o magneto para o compósito contendo 5\% de maghemita.

$$
F m=F_{\infty}+a_{1} \times e^{\frac{d}{a_{2}}}
$$

Na Equação 2, Fm é a força magnética; $F_{\infty}$ é a força que o imã exerce sobre a amostra com a distância entre elas tendendo ao infinito; $a_{1}$ é a amplitude; $a_{2}$ é a constante de decaimento e $d$ é a distância entre o centro do magneto e a amostra.

Os dados obtidos com o processo de modelagem e as suas respectivas correlações são mostrados naTabela5. Os modelos obtidos apresentaram correlações muito boas com os dados experimentais (Tabela 5). Esses modelos também permitem a extrapolação da força magnética para uma distância zero, denominada força magnética inicial $\left(F m_{0}\right)$, entre as amostras e o magneto. Nesta condição, a força magnética corresponde à soma dos parâmetros $F_{\infty}$ e $a_{1}$. Os valores calculados de $F m_{0}$ para o compósito e para a maghemita são iguais a $(2.0 \pm 0.4) \times 10^{-2}$ e $(9 \pm 8) \times 10^{-3} \mathrm{~N}$, respectivamente. Desta forma, os resultados obtidos mostram que as nanopartículas de maghemita dispersas na matriz polimérica, em comparação com a maghemita pura, são atraídas pelo imã com uma força 111\% maior. Este resultado está relacionado com a obtenção de um elevado grau de dispersão das partículas no material compósito, o que permite um aumento de sua capacidade de atração, o que é corroborado pelos resultados de SAXS.

Entre as possibilidades de uso deste material, uma de grande impacto está relacionada com a recuperação ambiental. Os testes utilizando o compósito PU-5 na remoção de petróleo derramado sobre água (Figura 7) mostraram que cada grama do compósito foi capaz de remover em média $(4,1 \pm 0,1) \mathrm{g}$ de petróleo. No intervalo testado, a massa de petróleo removida é linearmente proporcional à massa de resina utilizada, permitindo a construção de um modelo empírico, mostrado na Equação 3.

$$
\mathrm{PR}=-(1.81 \pm 2.19) \times 10^{-3}+(4.1 \pm 0.1) \times 10^{0} \times \mathrm{Res}
$$

Na Equação 3, PR é a quantidade de petróleo removido e Res é a quantidade de resina utilizada.

Assim, uma pequena porção de maghemita incorporada ao poliuretano promove uma atração magnética forte o bastante para que ocorra a total remoção da massa composta pelo compósito e pelo petróleo. Esses resultados são estimulantes uma vez que agregam recursos renováveis provenientes da indústria do biodiesel com a questão da recuperação ambiental. Isto conduz uma nova perspectiva quanto à recuperação de ambientes degradados bem como apresenta uma nova alternativa para a utilização do óleo de mamona. 
Tabela 5. Parâmetros da Equação 2 obtidos por mínimos quadrados.

\begin{tabular}{ccc}
\hline Parâmetro & \multicolumn{2}{c}{ Amostra } \\
\cline { 2 - 3 } & PU-5 & Maghemita \\
\hline $\mathrm{F}_{\infty}$ & $(3,4 \pm 0,4) \times 10^{-5}$ & $(3 \pm 2) \times 10^{-6}$ \\
$\mathrm{a}_{1}$ & $(2,0 \pm 0,4) \times 10^{-2}$ & $(9 \pm 8) \times 10^{-3}$ \\
$\mathrm{a}_{2}$ & $(7,6 \pm 0,3) \times 10^{-1}$ & $(1,2 \pm 0,3) \times 10^{0}$ \\
$\mathrm{R}^{2}$ & 0,9997 & 0,9959 \\
\hline
\end{tabular}

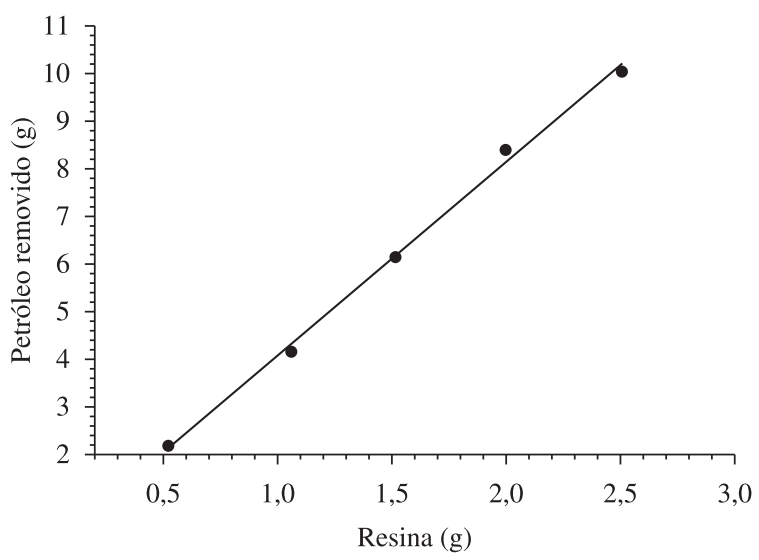

Figura 7. Teste de remoção de petróleo utilizando compósito de poliuretano magnetizável.

\section{Conclusões}

Um novo material magnético e ecologicamente correto foi preparado. Este material agrega as propriedades magnéticas da maghemita com a baixa densidade de materiais espumados provenientes da reação entre o óleo de mamona e o diisocianato de tolueno. Este material magnético apresenta considerável capacidade de atração e adsorção de petróleo na sua superfície, o que permite o seu uso na remoção de petróleo em ambientes aquáticos.

\section{Agradecimentos}

Os autores agradecem ao Conselho Nacional de Desenvolvimento Científico e Tecnológico (CNPq), à Fundação Carlos Chagas Filho de Amparo à Pesquisa do Estado do Rio de Janeiro (FAPERJ) e à Coordenação de Aperfeiçoamento de Pesquisa de Nível Superior (CAPES) pelo suporte financeiro e pelas bolsas. Os autores também agradecem ao Laboratório Nacional de Luz Síncrotron (LNLS) pelas análises de SAXS (LNLS Brasil - D11A -SAXS1 \# 7086/08 e 9077/10) e às alunas Jéssica Marins e Ana L. C. Ramos pelos ensaios de FTIR.

\section{Referências Bibliográficas}

1. Griffith, L. G. - Acta Mater., 48, p.263 (2000).

2. Souza Jr., F. G.; Richa, P.; Siervo, A.; Oliveira, G. E.; Rodrigues, C. H. M.; Nele, M. \& Pinto, J. C. - Macromol. Mater. Eng., 293, p.675 (2008).

3. Silva, R. V. - "Compósito de resina poliuretano derivada de óleo de mamona e fibras vegetais”, Tese Doutorado, Universidade de São Paulo, Brasil (2003).

4. Sharma, V. \& Kundu, P. P. - Progr. Polym. Sci., 33, p.1199 (2008).

5. Freire, R. M. M.; Severino, L. S. \& Machado, O. L. T. - "Ricinoquímica e co-produtos", in: O Agronegócio da mamona no Brasil, cap. 13,
Azevedo, D. M. P.; Beltrão, N. E. M. (Ed.), Embrapa Informação Tecnológica, Brasília (2006).

6. Woods, G. - "The ICI Polyurethanes book", John Wiley, New York (1990).

7. Bouvier, D. - Composites, 20, p.66 (1997).

8. Kawasumi, M.; Hasegawa, N.; Kato, M. \& Usuki, A. - Macromolecules, 30, p.6333 (1997).

9. Gnanaprakash, G.; Mahadevan, S.; Jayakumar, T.; Sundaram, P. K.; Philip, J. \& Raj. B. - Mater. Chem. Phys., 103, p.168 (2007).

10. Hong, R.Y.; Fenga, B.; Chena, L. L.; Liuc, G. H.; Li, H. Z.; Zheng, Y. \& Wei, D. G. - Biochem. Eng. J., 42, p.290 (2008).

11. Alexandre, M. \& Dubois, P. - Mater. Sci. Eng., 53, p.1 (2000).

12. Tjong, S. C. - Mater. Sci. Eng., 53, p.73 (2006).

13. Shen, Y. F.; Tang, J.; Nie, Z. H.; Wang, Y. D.; Ren, Y. \& Zuo, L. Bioresour. Technol., 100, p.4139 (2009).

14. Morales, M. A.; Jain, T. K.; Labhasetwar, V. \& Leslie-Pelecky, D. L. - J. Appl. Physi., 97, p.10905 (2005).

15. Choi, B. J. \& Leea, G. H. - J. Appl. Physi., 302, p.102-104 (2007).

16. Ahn, V. V.; Polichtchouk, Y. M. \& Yashchenko, I.G. - Organic Geochemistry, 33, p.1381 (2002).

17. Chang, C. L.; Fogler, H. S. - Fuel Sci. Tech., 14, p.75 (1996).

18. Oliveira, G. E. - "Comportamento de fases de parafinas, asfaltenos e ácidos naftênicos de petróleo e influência da presença de aditivos poliméricos", Tese de Doutorado, IMA, UFRJ, Brasil (2006).

19. Thomas, J. E.; Triggia, A. A.; Correia, C. A.; Verotti Filho, C.; Xavier, J. A. D.; Machado, J. C. V.; Souza Filho, J. E.; Paula, J. L.; Rossi, N. C. M.; Pitombo, N. E. S.; Gouvêa, P. C. V. M.; Carvalho, R. S. \& Barragam, R. V. - "Fundamentos de Engenharia de Petróleo", 2 ed., Interciência, Rio de Janeiro (2004).

20. CONAMA. - "CONAMA n 393: Resolução do conselho nacional do meio ambiente", Brasília (2007).

21. Qu, S.; Yang, H.; Ren D.; Kan S.; Zou G.; Li D. \& Li M. - J. Colloid Interface Sci., 215, p.190 (1999).

22. Kellermann, G.; Vicentin, F.; Tamura, E.; Rocha, M.; Tolentino, H.; Barbosa, A.; Craievich, A. \& Torriani, I. - J. Appl. Cryst., 30, p.880 (1997).

23. Souza Jr., F. G.; Soares, B. G. \& Dahmouche, K. - J. Polym. Sci. Part B, Polym. Phys., p.3069 (2007).

24. Lopes, M. L.; Oliveira, G. E. \& Souza Jr, F. G. - "Estudo da morfologia de híbridos de maghemita e polianilina usando AFM. 20a Reunião Anual de Usuários do LNLS" (2010).

25. Carneiro, A. A. O.; Touso, T. A. \& Baffa, O. - Quim. Nova, 26, p. 95 (2003).

26. Gyergyek, S.; Huskic, M.; Makovec, D. \& Drofenik, M. - Colloids Surf. A, Physicochem. Eng. Asp., 49-55, p.317 (2008).

27. Scherrer, P. - "Nachrichten Gesellschaft der Wissenschaften zu Göttingen" (1918).

28. Ruland, W. - Acta Cryst., 14, p.1180 (1961).

29. Souza Jr., F. G.; Marins, J. A.; Pinto, J. C.; Oliveira, G. E.; Rodrigues, C. M. \& Lima, L. M. T. R. - Journal of Material Science, Special Issue, p.1 (2010).

30. Nedkova, I.; Merodiiskaa, T.; Slavova, L.; Vandenbergheb, R. E.; Kusanoc, Y. \& Takadad, J. - J. Magn. Magn. Mater, 300, p.358 (2006).

Enviado: $14 / 04 / 10$

Reenviado: 09/06/10

Aceito: 16/06/10

DOI: $10.1590 / \mathrm{S} 0104-14282010005000054$ 